\title{
Solar and seasonal dependence of ion frictional heating
}

\author{
J. A. Davies ${ }^{1}$, M. Lester ${ }^{1}$ and I. W. McCrea ${ }^{2}$ \\ ${ }^{1}$ Radio and Space Plasma Physics Group, Department of Physics and Astronomy, University of Leicester, \\ University Road, Leicester, LE1 7RH, UK \\ ${ }^{2}$ EISCAT Group, Space Science Division, Rutherford Appleton Laboratory, Chilton, Didcot, Oxfordshire, OX11 0QX, UK
}

Received: 14 April 1998 / Revised: 5 October 1998 / Accepted: 20 October 1998

\begin{abstract}
Ion frictional heating constitutes one of the principal mechanisms whereby energy, originating in the solar wind, is deposited into the Earth's ionosphere and ultimately the neutral atmosphere. Common programme observations by the EISCAT UHF radar system, spanning the years 1984 to 1995 , provide the basis for a comprehensive statistical study of ion frictional heating, results of which are documented in this and a previous paper by the authors. In the present work, the authors demonstrate the solar and seasonal dependence of the universal time distribution of frictional heating, and explain these results with reference to corresponding dependences of the ion velocity. Although EISCAT observes a significant increase in the occurrence of enhanced ion velocities associated with increased solar activity, the latter characterised according to the prevailing $10.7 \mathrm{~cm}$ solar flux, this is not reflected to such an extent in the occurrence of frictional heating. It is suggested that this is a consequence of the decreased neutral atmosphere response times associated with active solar conditions, resulting from the higher ionospheric plasma densities present. Seasonal effects on the diurnal distribution of ion frictional heating are well explained by corresponding variations in ionospheric convection, the latter principally a result of geometrical factors. It is noted that, over the entire dataset, the variations in the unperturbed F-region ion temperature, required to implement the identification criterion for ion heating, are highly correlated with model values of thermospheric temperature.
\end{abstract}

Key words. Ionosphere (auroral ionosphere; ionosphere-atmosphere interactions; plasma temperature and density).

\section{Introduction}

Auroral electric fields, a manifestation of magnetosphere-ionosphere coupling, drive the F-region plasma through the neutral atmosphere thereby heating the ion population through collisions with thermospheric particles in a process termed ion frictional heating (see, e.g. Rees and Walker, 1968; Schunk et al., 1975). These collisions, however, tend to equalise the velocities of the two populations, in a mechanism referred to as ion drag (e.g. Rishbeth, 1972). The ion drag force on the neutral atmosphere is proportional to the electron density and, as such, is highly variable (Baron and Wand, 1983).

Theory predicts that a relative velocity between the ions and neutrals in the F-region should result in the distortion of the ion thermal velocity distribution from an isotropic Maxwellian, tending towards a torus in velocity space although remaining gyrotropic with respect to the magnetic field. Ion temperature anisotropy is, in consequence, predicted to develop relative to the field, with the field-perpendicular ion temperature exceeding the temperature of the field-parallel ion population (e.g. St-Maurice and Schunk, 1977, 1979). Experimental evidence indicating the existence of nonMaxwellian line-of-sight ion velocity distributions and anisotropic ion temperatures has been provided both by satellites (e.g. St-Maurice et al., 1976) and radar observations (e.g. Perraut et al., 1984; Lockwood et al., 1987).

Solution of the ion energy balance equation, simplified appropriate to F-region conditions, yields the following expressions for the steady-state enhancement in the field-parallel and field-perpendicular components of the ion temperature in response to frictional heating the temperature components themselves are denoted by $T_{i \|}$ and $T_{i \perp}$ respectively.

$T_{i \|}-T_{e q}=\frac{\left\langle m_{n}\right\rangle}{2 k_{B}} \beta_{\|}\left(\mathbf{v}_{i}-\mathbf{v}_{n}\right)^{2}$ 
$T_{i \perp}-T_{e q}=\frac{\left\langle m_{n}\right\rangle}{2 k_{B}} \beta_{\perp}\left(\mathbf{v}_{i}-\mathbf{v}_{n}\right)^{2}$

in which $\left\langle m_{n}\right\rangle$ represents the mean neutral mass, $k_{B}$ is Boltzmann's constant, $\mathbf{v}_{i}$ and $\mathbf{v}_{n}$ represent the ion and neutral velocities and the field-parallel and field-perpendicular ion temperature partition coefficients are denoted by $\beta_{\|}$and $\beta_{\perp}$, respectively (e.g. St-Maurice and Schunk, 1977). Such steady state conditions are attained in a characteristic time which is the inverse of the ionneutral collision frequency, of the order of seconds. The unperturbed ion temperature, $T_{e q}$, defined as the ion temperature in the absence of frictional heating, is equivalent to the sum of the neutral temperature and a small contribution due to electron-ion thermal energy exchange.

In a recent paper, Davies et al. (1997) (see also Davies et al., 1998) presented results from a statistical study of ion frictional heating, based on over $4000 \mathrm{~h}$ of common programme 1 (CP-1) and common programme 2 (CP-2) observations taken by the EISCAT UHF incoherent scatter radar between October 1984 and December 1995. In addition to deriving the diurnal distribution of ion frictional heating, Davies et al. (1997) (hereafter referred to as paper A) investigated its dependence on both the orientation of the interplanetary magnetic field and geomagnetic activity. The present work is an extension to paper A; here the authors further assess the seasonal dependence of ion frictional heating and also its dependence on solar activity, since the observations extend over an entire solar cycle, solar cycle 22.

\section{Experimental arrangement}

The EISCAT UHF incoherent scatter radar system, which operates at frequencies around $933 \mathrm{MHz}$, comprises three parabolic dish antennas, one sited at Tromsø, Northern Norway, which combines both transmit and receive capabilities, and two remote site receivers, at Kiruna, Sweden, and Sodankylä in Finland. Notwithstanding the ability to measure those ionospheric parameters, such as ion temperature and electron density, which is standard amongst incoherent scatter radars, the unique tristatic nature of the EISCAT UHF radar allows the full ion vector velocity to be determined within the intersection volume of the three receiver beams.

The following work is based on observations taken by the UHF radar in common programme $1(\mathrm{CP}-1)$ and common programme 2 (CP-2) modes. The geometrical arrangement of both experiments and their measurement techniques are discussed in detail in paper A, and, as such, are summarised only briefly here.

In CP-1, the UHF transmitter beam is aligned along the local magnetic field direction in the F-region which, for a radar located at Tromsø, is at an elevation of around $77^{\circ}$ and an azimuth of $182^{\circ}$. In CP-2, however, the transmitter performs a four position scan, in which one position is field-aligned, with a total scan time of 6 min. In both experiments, the remote site radars provide F-region tristatic observations near $300 \mathrm{~km}$ altitude, although, incidentally, in those versions of CP1 employed in this study the remote site radars also scanned through the E-region.

The long pulse measurements, which are the basis for the present study, yield an altitude resolution of some $22 \mathrm{~km}$ along the magnetic field direction, from approximately 150 to $600 \mathrm{~km}$ altitude; high resolution observations at E-region altitudes are provided by either multi-pulse or alternating code transmission. CP-1 observations from Tromsø have been post-integrated at a temporal resolution of 5 minutes and the remote site data over the dwell time at each scan position. CP-2 observations from all sites are post-integrated over the dwell time in each scan position, some $90 \mathrm{~s}$.

\section{Selection criterion for the identification of ion frictional heating}

The criterion adopted by the present authors, and used previously in paper $\mathrm{A}$, for the identification of ion frictional heating was that initially devised by McCrea et al. (1991). A detailed explanation of the selection criterion, and the rationale behind it, is presented by both McCrea et al. (1991) and in paper A and, as such, will not be repeated in full here. Instead, the selection criterion is simply stated.

Identification of an interval of ion frictional heating requires an enhancement in the ion temperature equal to or exceeding $100 \mathrm{~K}$, for two or more consecutive integration periods. Analysis is constrained to fieldparallel observations from the long-pulse range gate centred at the F-region altitude of $312 \mathrm{~km}$. Implementation of the selection criterion relies on the estimation of an unperturbed ion temperature for which the modal value of the distribution of the ion temperature, calculated at $312 \mathrm{~km}$ altitude for each experiment run with a bin width of $10 \mathrm{~K}$, is considered appropriate; runs have a typical duration of between 24 and $48 \mathrm{~h}$.

An enhancement in the F-region field-parallel ion temperature of $100 \mathrm{~K}$, through frictional heating, requires an ion velocity of around $500 \mathrm{~m} \mathrm{~s}^{-1}$, though this assumes a stationary neutral atmosphere. To assist in the interpretation of the statistics of frictional heating, following the work documented in paper A, this threshold is implemented in order to identify enhancements in the UHF tristatic ion velocities measurements. In order to eliminate unreliable estimates of ion velocity, any velocity estimates for which the signal-to-noise ratio at any of the three receiver sites was less than $2 \%$ are excluded.

\section{Statistical study of ion frictional heating}

\subsection{Application of the selection criterion}

The selection criterion for the identification of ion frictional heating, stated in the previous section, was applied to the present extended data set of CP-1 and field-aligned CP-2 long-pulse observations of field-paral- 
lel ion temperature at $312 \mathrm{~km}$ altitude. The variation over the $12 \mathrm{y}$ data set of the unperturbed ion temperature at $312 \mathrm{~km}$, for which the modal ion temperature calculated as described in the previous section is used as an approximation, is illustrated in Fig. 1 (solid line). Also illustrated, represented by a dotted line, is the temperature of the neutral atmosphere calculated for the same altitude and at the location of EISCAT, using the MSIS-90 model (Hedin, 1991). The value of neutral temperature presented is the mode calculated for the first day of each month. The values of the $A_{P}$ index and the $10.7 \mathrm{~cm}$ solar flux $\left(\mathrm{F}_{10.7}\right)$, input parameters required by the MSIS model to characterise geomagnetic and solar conditions, were those appropriate to the days for which the model was run. The neutral and ion temperatures exhibit a similar trend, both seasonally and with solar cycle, tending to be highest in summer and towards the peak of the solar cycle, corresponding to increased solar UV and X-ray photon flux. In general, the unperturbed temperature of the ion population tends to exceed slightly the neutral temperature, indicative perhaps of energy transfer to the ions from the hotter electron gas. The difference in the unperturbed ion temperature between conditions of solar minimum and solar maximum is some $500 \mathrm{~K}$ while its annual variation is less then $300 \mathrm{~K}$. The effect of frictional heating on the ion temperature often exceeds that attributable to solar cycle and seasonal variations, illustrating the importance of ion frictional heating as a source of energy deposition into the high-latitude ionosphere and thermosphere.

A total of 1152 intervals of ion frictional heating were identified from the 4230 hour set of field-parallel ion temperature observations; indeed frictional heating was observed to some extent during the vast majority of the EISCAT common programme runs incorporated into the study. The diurnal distribution of ion frictional heating was presented in paper A, having been derived from the entire data set by dividing the day into $30 \mathrm{~min}$ bins and summing the number of occasions over the dataset on which heating occurred in each bin. To negate the effect on non-uniform sampling, the distribution was expressed as a percentage of the number of observations of each bin. Similarly, to aid interpretation, the distribution of enhanced ion velocities was constructed. These two distributions, along with a physical explanation of their forms, are discussed in great detail in paper A and, because of their relevance to the present work in which they are subdivided according to solar and seasonal factors, are summarised here.

The diurnal distribution of ion frictional heating, presented in paper A, exhibits a broad peak in occurrence in the night-time sector with a much reduced incidence of frictional heating between some 06:00 and 13:00 Universal Time (UT) on the dayside (at EISCAT, Local Time $(\mathrm{LT})=\mathrm{UT}+1 \mathrm{~h}$ and Magnetic Local Time $(\mathrm{MLT}) \sim \mathrm{UT}+2 \mathrm{~h}$ ). This reduced occurrence of frictional heating during the daytime hours is a consequence of the equatorward edge of the ionospheric convection pattern typically being situated poleward of the radar during these hours, a result of the displacement of the convection pattern as a whole in the antisunward direction. This interpretation is supported by the occurrence of a corresponding nightside peak in the distribution of enhanced ion velocities. The diurnal distribution of ion frictional heating is centred at approximately 20:00 UT (22 MLT) manifesting a nightside minimum about which the distribution is asymmetric with a propensity for frictional heating in the early morning sector. This nightside minimum corresponds to an interval extending over several hours, in the region of the nightside convection reversal, during which the ion velocities tend to be of depressed magnitude and fluctuate in direction, as confirmed by observations of electric field, at comparable magnetic latitudes, by the incoherent scatter radar at Chakanika, Alaska (Banks and Doupnik, 1975). The dawn-dusk asymmetry in the occurrence of frictional heating, a feature which is not evident in the distribution of enhanced ion velocity, is consistent with observations by Baron and Wand (1983), Alcaydé et al. (1984), Alcaydé

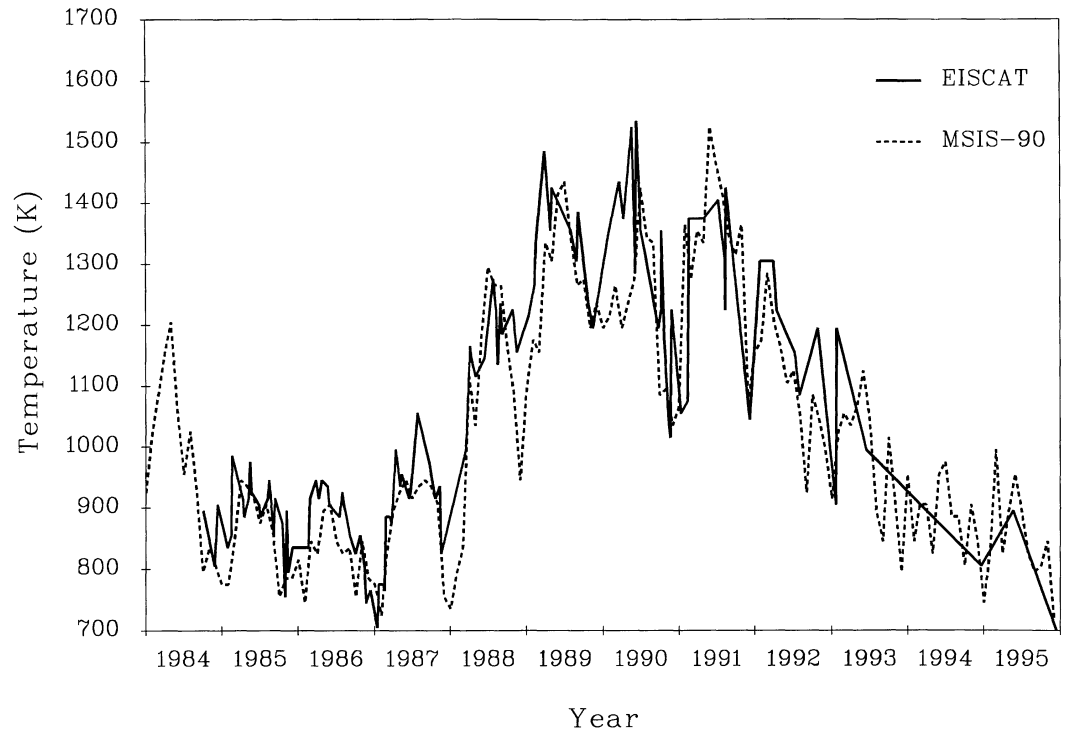

Fig. 1. Variation of the unperturbed ion temperature, derived from the EISCAT CP-1 and CP-2 observations at $312 \mathrm{~km}$ altitude (solid line). The dotted line indicates the variation of the thermospheric temperature at the same altitude, from the MSIS-90 model 
and Fontanari (1986) and Moorcroft and Schlegel (1988) of larger ion temperatures in the post-midnight sector and results from a tendency for smaller neutral winds to develop in the post-midnight sector than prior to magnetic midnight, an effect to which several processes are thought to contribute.

The F-region electron density is generally lower in the morning sector than during the afternoon and evening hours, due to the decay of solar produced ionisation, thus the ion drag force on the neutral atmosphere, which is proportional to the plasma concentration, will tend to be less. This weaker coupling between the ions and the neutral population post midnight, results in larger ionneutral relative velocities and, in consequence, more intense ion frictional heating during these hours (Baron and Wand, 1983). Another factor which determines the extent to which the neutral wind can be accelerated by ion drag is the length of time for which it is resident within the region of large auroral ion flows. In the dusk convection cell, where opposing Coriolis and centrifugal forces tend to cancel, neutral air tends to remains within the region of strong ion flows for longer than in the dawn cell, where both forces act to move the air equatorward out of the oval preventing it from acquiring a large momentum from the ions (e.g. Lockwood and Fuller-Rowell, 1987a, b). In a similar vein, Alcaydé et al. (1984) suggested that the strongly developed southward neutral winds which exist on the nightside as a feature of global thermospheric circulation, would induce a Coriolis force which opposes the ion drag force arising from eastward plasma flow during the postmidnight hours whereas in the evening sector these two forces would act in the same direction.

It is pertinent to remark that increasing the enhancement necessary for the identification of frictional heating to $200 \mathrm{~K}, 300 \mathrm{~K}$ or, indeed, higher does not significantly alter the shape of its distribution. This confirms that the application of a threshold enhancement of $100 \mathrm{~K}$ does not lead to the selection of extraneous intervals of frictional heating caused by normal diurnal variations.

\subsection{Variation with solar activity}

The set of EISCAT common programme observations were loosely classified according to prevailing solar conditions into those taken during low solar activity $\left(\mathrm{F}_{10.7}<150\right)$ and high solar activity $\left(\mathrm{F}_{10.7} \geq 150\right)$. Figure 2 illustrates the diurnal distribution of ion frictional heating observed by EISCAT at $312 \mathrm{~km}$ altitude for these two ranges of solar activity. The histograms represents the percentage occurrence of ion frictional heating with universal time (left hand scale) while the superimposed lines plots illustrate the number of observations of each $30 \mathrm{~min}$ bin (right hand scale). Figure 3 presents equivalent distributions of enhanced F-region ion velocities, those with a magnitude exceeding $500 \mathrm{~m} \mathrm{~s}^{-1}$. Velocities are further categorised in terms of the orientation of their associated zonal component; ion velocities in which the zonal component is westward are represented by light shading whereas darker shading illustrates ion velocities with an associated eastward component.

The distributions presented in Figs. 2 and 3 are not significantly different, in shape, to those derived from the entire data set, as presented in paper A, although binning the data with respect to conditions of solar activity does affect the observed incidence of frictional heating and enhanced ion velocity, particularly in the case of the latter. It is, indeed, instructive initially to consider the variation of the diurnal distribution of high ion flows with solar activity. It is evident from

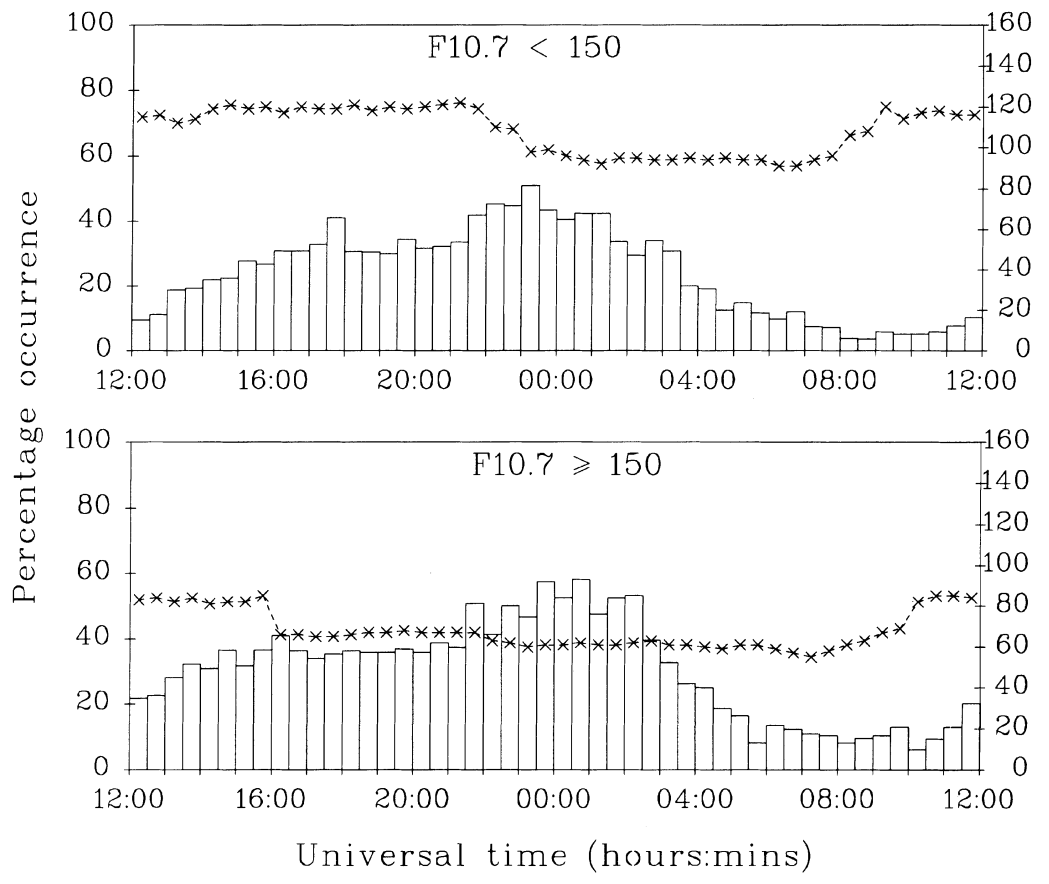

Fig. 2. Variation of the diurnal distribution of ion frictional heating at $312 \mathrm{~km}$ altitude with solar activity, characterised according to $\mathrm{F}_{10.7}$. The histograms represent the percentage occurrence of frictional heating as a function of universal time (left hand scale); the line plots indicate the number of observations of each half hour bin (right hand scale) 


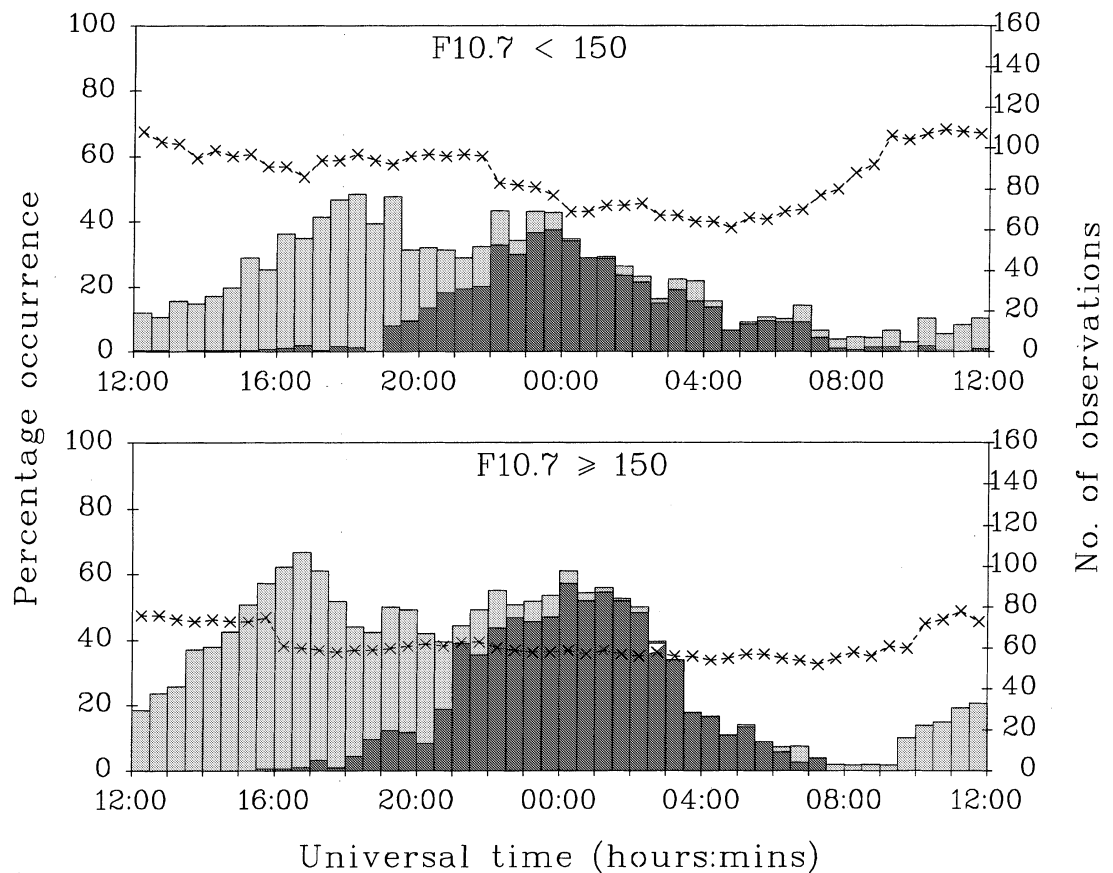

Fig. 3. Variation of the diurnal distribution of enhanced F-region ion velocity with solar activity. The histograms represent the percentage occurrence of enhanced ion flow as a function of universal time (left hand scale); light grey shading indicates enhanced ion velocities with a westward zonal component, dark grey indicates those with an eastward zonal component. The line plots, again, indicate the number of observations of each half hour bin (right hand scale)
Fig. 3 that the occurrence probability of ion velocities exceeding $500 \mathrm{~m} \mathrm{~s}^{-1}$ in magnitude tends to increase markedly at all universal times with higher solar activity. Near 00:00 UT, for example, the percentage occurrence of enhanced ion velocity is around $40 \%$ for low solar activity, rising to more than $60 \%$ under active solar conditions, although there is no significant difference in the form of the two distributions. This trend can be explained with respect to the solar cycle variation of the geomagnetic activity. Magnetic activity on the Earth tends to increase with increasing solar activity, characterised by a greater incidence of disturbances on the Sun, such as solar flares and coronal holes. A study by Hapgood et al. (1991), based on satellite observations taken over two complete solar cycles (numbers 20 and 21), revealed a distinct solar cycle variation in the IMF; the authors noted that the $z$ component of the IMF tends to be of larger magnitude, and display a greater variance, at sunspot maximum, a factor which will contribute, at least in part, to a corresponding solar variation in geomagnetic activity. The median values of the $K_{p}$ index corresponding to low and high solar activity as defined in this study are $2+$ and 3-, respectively. This difference may seem minimal but paper A illustrates the significant increase between the probability of observing enhanced F-region plasma flows for values of $K_{p}$ around 2 and that for values of $K_{p}$ around 3, from less than $40 \%$ to almost $70 \%$ for a half hour bin around midnight UT. A solar cycle variation in F-region ion velocities measured by the EISCAT UHF radar has previously been demonstrated by Aruliah et al. (1996) who found that, in general, the average velocities were significantly higher around solar maximum except in winter when the ion velocities were slightly greater at solar minimum.
The diurnal distribution of ion frictional heating (Fig. 2) does not exhibit nearly such a marked difference between conditions of low and high solar activity as that observed in the ion velocities, although, on average, the occurrence of frictional heating does still increase with ascending solar activity. More quantitatively, the average percentage occurrence of ion frictional heating, averaged over the entire day, is only some $25 \%$ more on solar active days whereas, for enhanced ion velocities this increase is more than $50 \%$. The implication is of a mechanism which acts to suppress ion frictional heating preferentially at times of high solar activity or, equivalently, enhance frictional heating during low solar activity. Further analytical evidence, exploiting the ability of the radar to make simultaneous measurements of ion temperature and velocity, illustrates more clearly the suppression of frictional heating around solar maximum. Figure 4 presents the field-parallel ion temperature enhancement as a function of ion velocity during identified intervals of ion frictional heating. Indicated are the median enhancements in consecutive velocity bins of $100 \mathrm{~m} \mathrm{~s}^{-1}$ width for both conditions of high $\left(\mathrm{F}_{10.7} \geq 150\right.$ : dotted line) and low solar activity $\left(\mathrm{F}_{10.7}<150\right.$ : solid line), with vertical lines which indicate the range bounded by the upper and lower quartiles (offset for clarity). Also shown, in corresponding line styles, are the number of estimates of parallel temperature enhancement in each velocity bin. Under quiet solar conditions, an ion velocity of value $1000 \mathrm{~m} \mathrm{~s}^{-1}$ results in a median enhancement in the field-parallel ion temperature of some $400 \mathrm{~K}$ while under more active solar conditions the corresponding value is only $250 \mathrm{~K}$. For ion velocities around $1500 \mathrm{~m} \mathrm{~s}^{-1}$, corresponding values are 400 and $800 \mathrm{~K}$, respectively.

The factors which determine the extent of ion frictional heating in the field-parallel direction are the 


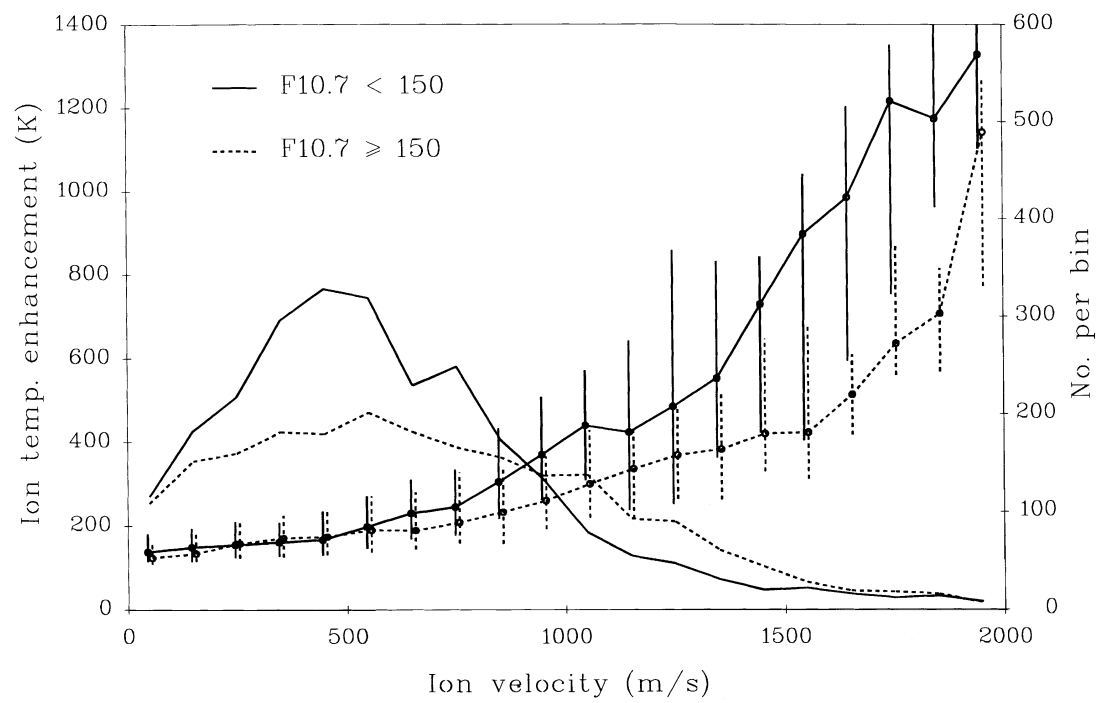

Fig. 4. Field-parallel ion temperature enhancement as a function of ion velocity during identified intervals of ion frictional heating. Indicated are the median enhancements in each $100 \mathrm{~m} \mathrm{~s}^{-1}$ width velocity bin for conditions of both high (dotted line) and low solar activity (solid line), with vertical lines which indicate the range bounded by the upper and lower quartiles. Also shown, in corresponding line styles, are the number of estimates of parallel temperature enhancement in each velocity bin mean ion mass, the field-parallel ion temperature partition coefficient and the ion-neutral difference velocity (see Eq. 1a). The first two would tend to increase the parallel ion temperature enhancement with increasing solar activity for a given ion flow velocity. Estimates of both of these quantities, based on the MSIS-90 model thermosphere, illustrate their tendency to increase towards solar maximum in response to composition and temperature changes in the neutral atmosphere, albeit by only a small amount compared with their seasonal variation (see Sect. 4.3). The apparent suppression of frictional heating towards active solar conditions cannot, therefore, be explained with reference to variations in the parallel ion temperature coefficient and the mean ion mass. It must be that, for a given ion velocity, the magnitude and direction of the neutral wind at solar maximum are such as to reduce the differential velocity between the two populations.

As noted previously, the ion and neutral populations are coupled through the force of ion drag (e.g. Rishbeth, 1972). The neutral velocity approaches the ion velocity exponentially with a time constant which is inversely proportional to the plasma density and directly proportional to the ratio of the neutral atmosphere mass density to the ion-neutral collision frequency (e.g. Baron and Wand, 1983). Results from the MSIS-90 model indicate that the latter varies only marginally with solar activity, decreasing by less than $5 \%$ between solar minimum and solar maximum. Although this would act to reduce the neutral response time under active solar conditions, leading to higher neutral winds for a given ion flow, it is clear that the effect would be far less than the effect on the ion drag time constant attributable to the solar cycle variation of the plasma density. The ionospheric plasma concentration exhibits, on average, an $11 \mathrm{y}$ periodicity corresponding to the variation of solar emission in the X-radiation and ultraviolet wave bands between solar minimum and solar maximum, resulting in, at least, a doubling of the electron density in the high-latitude F-region (e.g. Farmer et al., 1990). Plasma density effects will, therefore, decrease the response time of the neutral atmosphere substantially with increasing solar activity, permitting the generation of far larger neutral flows for a given ion flow speed and, consequently, suppressing frictional heating. These observations, indeed, highlight the significant influence of the electron density in determining, through ion drag, the extent of ion frictional heating through the solar cycle. This is an analogous effect to that proposed by Baron and Wand (1983) which is thought to contribute to the asymmetry in ion frictional heating between the pre and post-midnight sectors (see Sect. 4.1).

\subsection{Variation with season}

In order to ascertain any seasonal dependence of ion frictional heating, the EISCAT observations were subdivided according to season, with the seasons defined as centred on the appropriate equinoxes and solstices. Successive panels of Fig. 5 illustrate the diurnal distribution of ion frictional heating at $312 \mathrm{~km}$ altitude, derived from the EISCAT UHF long-pulse field-parallel CP-1 and CP-2 measurements, during winter, spring/ autumn, and summer respectively. As in previous figures of this type, the histograms represent the percentage occurrence of ion frictional heating as a function of universal time, with a bin width of $30 \mathrm{~min}$ extent (left hand scale) and the line plots illustrate the number of observations of each bin (right hand scale). Correspondingly, Fig. 6 illustrates the variation of the diurnal distribution of enhanced F-region ion velocity with season. The equinoctial distributions are incorporated into a single panel as they exhibit no discernible difference.

It is helpful, initially, to determine if the observations indicate any seasonal dependence in enhanced F-region ion flow at the location of EISCAT. Figure 6 reveals that there is a distinct seasonal variation in the diurnal distribution of large ion velocities, not just in the level of incidence but also in the form of the distribution compared to that of the general distribution presented 


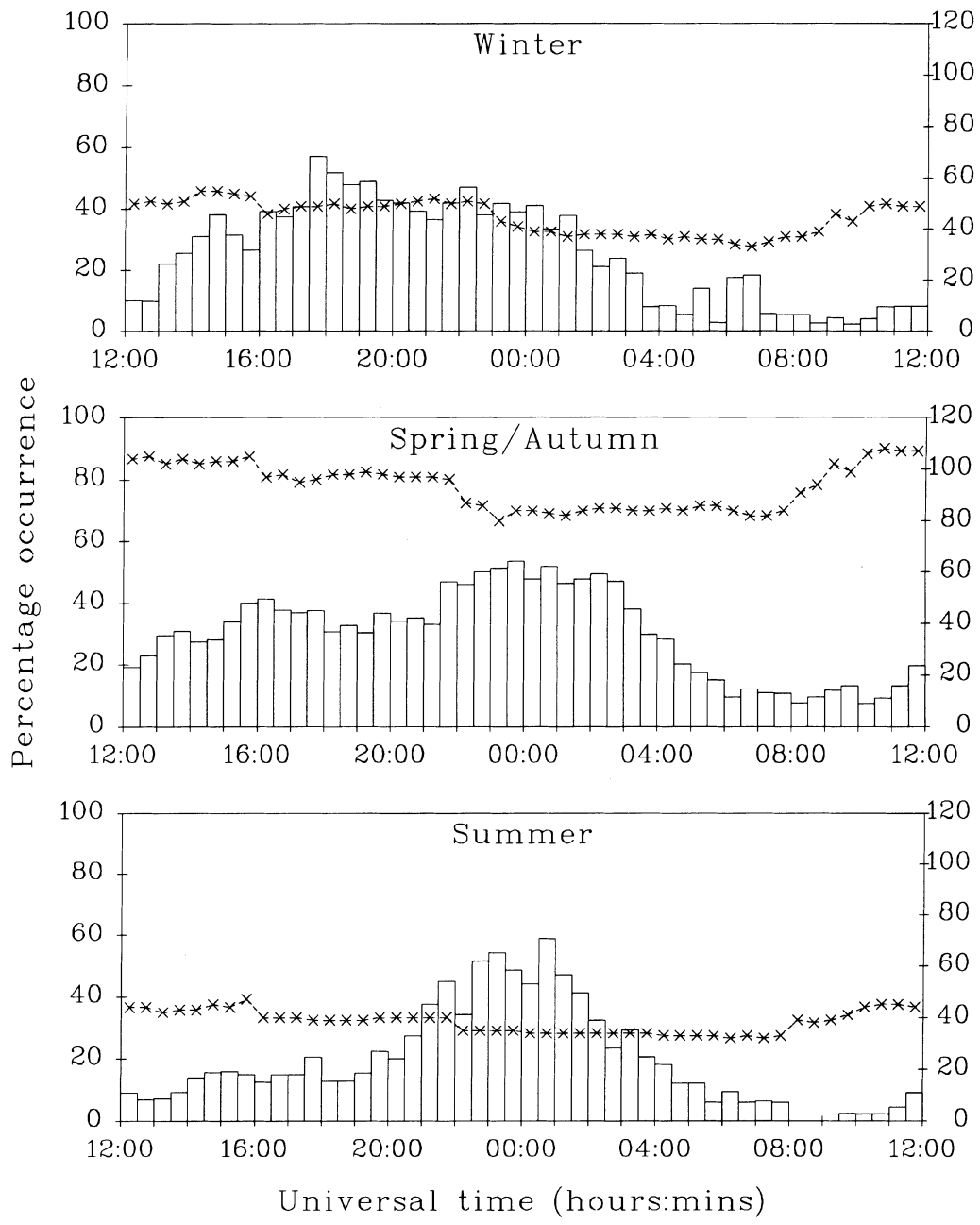

Fig. 5. Seasonal variation of the diurnal distribution of ion frictional heating at $312 \mathrm{~km}$. The format of each panel is as for Fig. 2 in paper A. Although for the equinoctial seasons there remains a roughly symmetric occurrence of large velocities in the dawn and dusk convection cells, this is not so for summer and winter. This asymmetry in the convection pattern between summer and winter, at least at the location of the radar, is manifested as a preponderance of enhanced ion flow in the pre-midnight hours during the winter months and, conversely, in the dawn convection cell during summer. In the post-midnight sector, the probability of observing ion velocities exceeding $500 \mathrm{~m} \mathrm{~s}^{-1}$ is comparable in summer and winter, but significantly greater during the equinoctial seasons. Before midnight, the incidence of such flows is by far the lowest during the summer months and slightly higher in winter than in spring/autumn. The average occurrence of enhanced ion velocities during the equinoctial seasons is some $20 \%$ higher than it is during the winter months and nearly double that in summer.

Previous authors have suggested that the convection pattern in the high-latitude ionosphere varies seasonally. Foster et al. (1986) and Holt et al. (1987) investigated the seasonal variation of convection although they could not obtain a complete description of seasonal dependence as their data, already subdivided according to IMF and $K_{p}$, was too sparse to draw any definitive conclusions. Later work by de la Beaujardière et al.
(1991) concentrated purely on seasonal variations in convection. The authors present average patterns of ionospheric convection between 67 and $82^{\circ}$ invariant latitude, derived for each season from observations taken between 1983 and 1988 by the Sondrestromfjord incoherent scatter radar. The authors de la Beaujardière et al. (1991) suggested that a major factor affecting ionospheric convection on a seasonal basis is the semiannual variation in geomagnetic activity. Geomagnetic conditions are most active at the equinoxes, a phenomenon proposed to result from a semi-annual variation in the effective southward component of the IMF caused by the seasonal variation in the Earth's dipole tilt angle (Russell and McPherron, 1973). The greater occurrence of enhanced ion velocities noted in the present study concur with the assertion that geomagnetic activity tends to be higher at the equinoxes and, indeed, over the EISCAT data set, the median value of $K_{p}$ is 3- for the equinoctial seasons, $2+$ for winter and 20 during the summer months. As illustrated in paper A, and mentioned previously with reference to the solar activity effects, even such a small variation in $K_{p}$ is associated with large changes in the intensity and extent of the convection pattern at the latitude of Tromsø. Furthermore, de la Beaujardière et al. (1991) suggested that, as the Earth's dipole tilt angle changes, so does the relative 

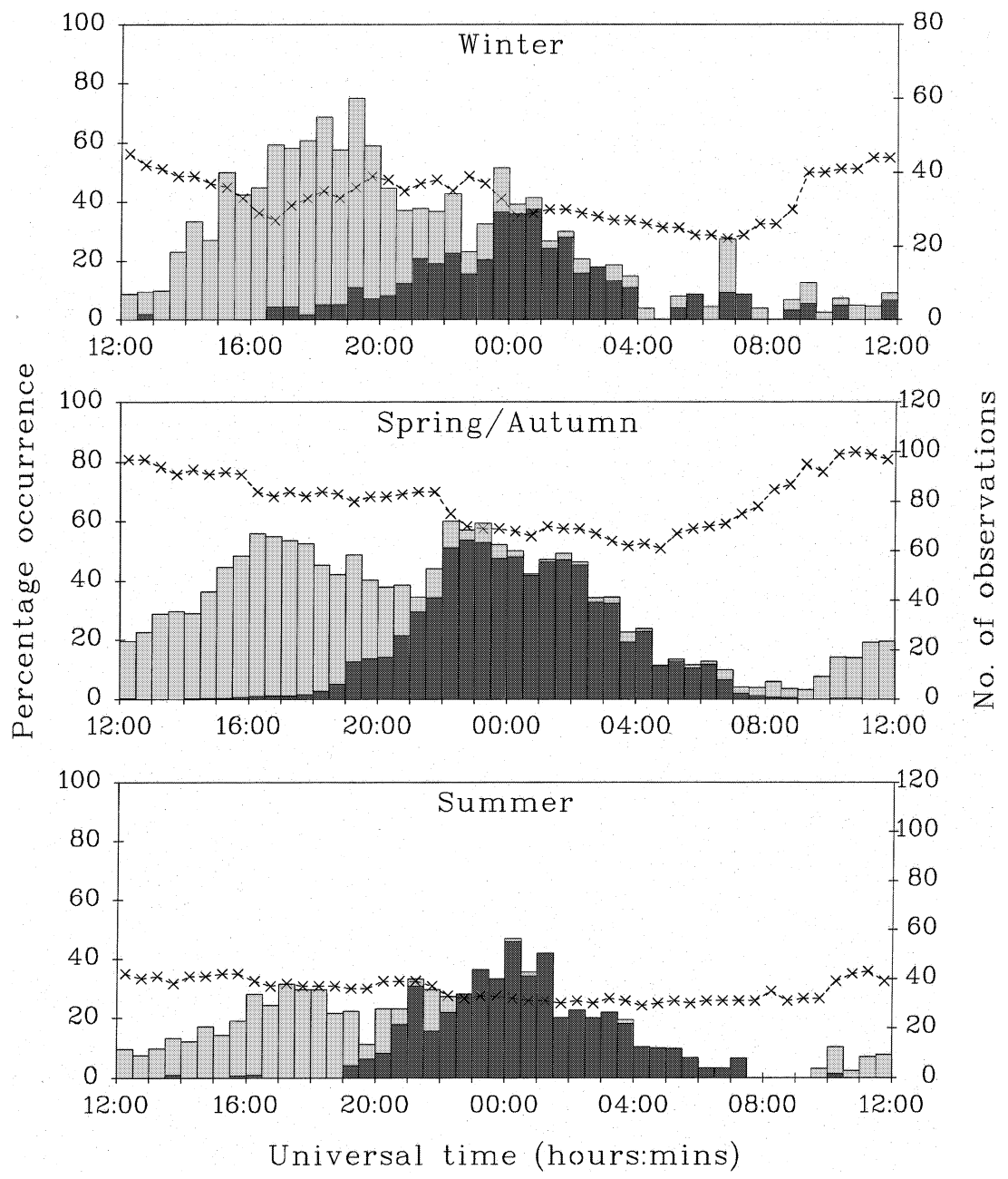

Fig. 6. Seasonal variation of the diurnal distribution of enhanced F-region ion velocity. The format of each panel is as for Fig. 3 position of the large-scale convection pattern, such that between summer and winter the pattern moves antisunward and also towards dawn. At the lower latitude boundary of the field of view of the Sondrestromfjord incoherent scatter radar, equivalent to the geomagnetic location of the present observations, de la Beaujardière et al. (1991) noted that larger velocities tend to arise before midnight in winter and during the post-midnight hours in summer, attributable to dipole tilt effects on the position of the large-scale convection pattern. Evidence for a winter/summer asymmetry is clearly supported by results of the present study, which reveals a far greater incidence of enhanced ion flow during the pre-midnight hours in winter and in the dawn sector in summer. As mentioned earlier, this asymmetry is not evident during spring and autumn.

The general shape of the diurnal distribution of ion frictional heating for each season (Fig. 5) is consistent with what would be anticipated from the corresponding distribution of enhanced ion velocity. During the equinoctial seasons, where there is an almost symmetric occurrence of enhanced ion flows in the dawn and dusk sectors, the tendency for larger ion-neutral relative velocities to develop during the early morning hours (see paper A) introduces an asymmetry in the distribution of ion frictional heating between these two regimes.
During summer, where the occurrence of enhanced velocities is less during the post-midnight hours, this asymmetry becomes more pronounced and in the winter months there is a slightly greater occurrence of frictional heating before midnight. The average occurrence of frictional heating, like that of high ion flows, is largest during the equinoctial seasons and least in summer.

Figure 7 illustrates the variation of the field-parallel ion temperature enhancement as a function of ion velocity during identified intervals of ion frictional heating for the different seasons. Indicated are the median enhancements in each $200 \mathrm{~m} \mathrm{~s}^{-1}$ width velocity bin for winter (solid line), the equinoctial seasons (dotted line) and summer (dashed line), again the vertical lines indicate the range bounded by the upper and lower quartiles. Also shown, in corresponding line styles, are the number of estimates of parallel temperature enhancement in each velocity bin. The median value of the field-parallel ion temperature for a given ion velocity is significantly higher in summer than in winter and the equinoctial seasons, at least for ion velocities over some $1400 \mathrm{~m} \mathrm{~s}^{-1}$, although for velocities less than this value, frictional heating appears to be equally effective regardless of season. Notably, even though the probability of observing ion frictional heating is least during summer, the effect of frictional heating on the ion 


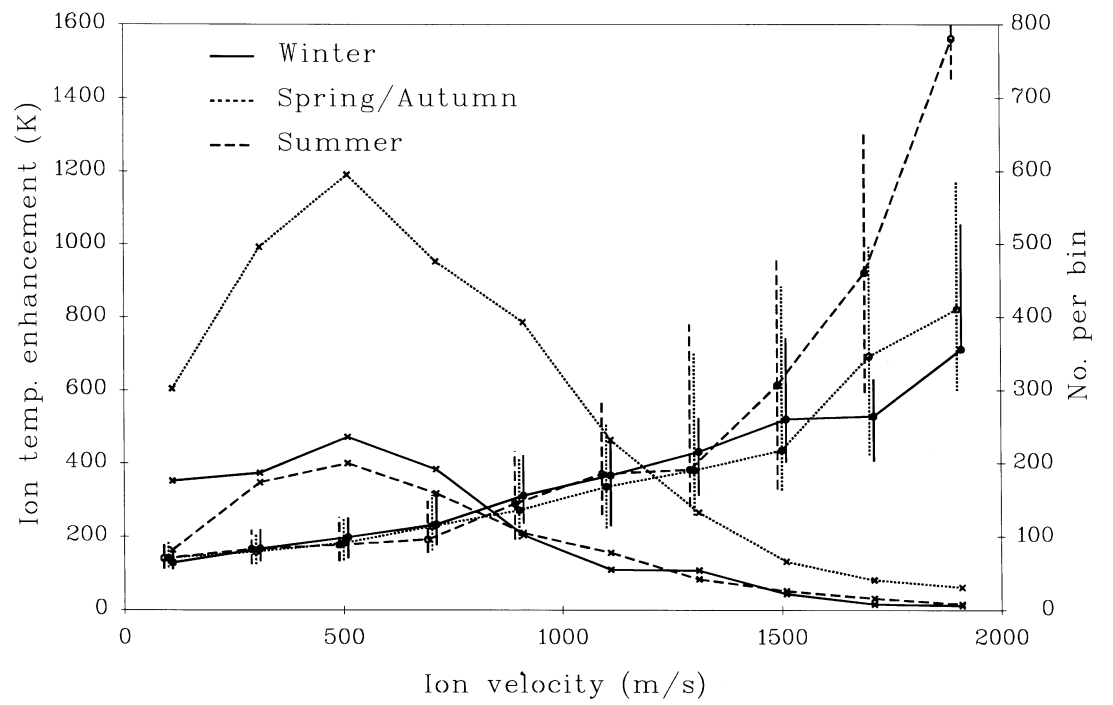

Fig. 7. Field-parallel ion temperature enhancement as a function of ion velocity during identified intervals of ion frictional heating. Indicated are the median enhancements in each $200 \mathrm{~m} \mathrm{~s}^{-1}$ width velocity bin for winter (solid line), the equinoctial seasons (dotted line) and summer (dashed line), with vertical lines which indicate the range bounded by the upper and lower quartiles. The number of estimates of parallel temperature enhancement in each velocity bin are illustrated in corresponding line styles temperature is actually greatest during these months. This result cannot be reconciled with the effect of seasonal variations of electron density on the neutral wind, through ion drag, since on the nightside, where EISCAT observes the vast majority of frictional heating, the electron density at F-region altitudes is a factor of two higher in summer than during the winter months, with intermediate values in the equinoctial seasons (Farmer et al., 1990). The exclusion of daytime events does not significantly alter Fig. 7 confirming that there is not a disproportionate effect from the few intervals of frictional heating on the dayside, where the winter anomaly means that the winter electron densities far exceed those during summer.

Model calculations using MSIS-90 predict significant increases in the mean neutral mass and the field-parallel ion temperature partition coefficient, by some $20 \%$ and $30 \%$ respectively, towards the summer months. Similarly, the ratio of the thermospheric mass density to the ion-neutral collision frequency which influences the ion drag time constant is predicted to increase markedly towards summer by around $20 \%$ compared to winter values (contrary to its slight decrease towards solar maximum). It is suggested that the effect of larger electron densities must be outweighed by increases in these three parameters, making ion frictional heating most effective in the summertime.

\section{Summary and main conclusions}

The current work, along with a previous paper by the authors, document results of a statistical analysis of Fregion ion frictional heating, based on an extensive set of observations by the EISCAT UHF radar, a database spanning the years 1984 to 1995 and comprising more than $4000 \mathrm{~h}$ of common programme observations. In this study, both the solar and seasonal dependence of the universal time distribution of frictional heating are demonstrated, and explained with reference to corresponding dependences of the ion velocity.
The general, yet only moderate, increase in the occurrence of ion frictional heating with increased solar activity, characterised according to the prevailing $10.7 \mathrm{~cm}$ solar flux, appears, at first sight inconsistent with the far more pronounced increase in the occurrence of enhanced ion velocities. It is suggested that this apparent suppression of frictional heating under conditions of increased solar activity is a consequence of the increased ion drag force on the neutral atmosphere resulting from the higher plasma densities present. This conclusion is supported by the analysis of simultaneous temperature and velocity measurements from the radar.

Seasonal variations in ionospheric convection, principally a result of geometric factors, provide an explanation for corresponding effects on the form of the diurnal distribution of ion frictional heating. However, the effect of seasonal variations in the neutral response time to ion drag, which may be expected to preferentially suppress frictional heating on the summer nightside, do not appear evident. Model calculations suggest that the effect of larger electron densities is outweighed by increases in both the mean neutral mass and the field-parallel ion temperature partition coefficient, such that ion frictional heating is actually most effective in raising the parallel ion temperature during summertime.

Acknowledgements. Thanks are due to the director of EISCAT and his staff for operating the facility and supplying the data. EISCAT is an association of the national scientific agencies of Finland, France, Germany, Japan, Norway, Sweden and the United Kingdom. J.A. Davies is supported by a grant from the Particle Physics and Astronomy Research Council.

Topical Editor D. Alcaydé thanks A. Brekke and another referee for their help in evaluating this paper.

\section{References}

Alcaydé, D., and J. Fontanari, Neutral temperature and winds from EISCAT CP-3 observations, J. Atmos. Terr. Phys., 48, 931, 1986. 
Alcaydé, D., P. Bauer, and J. Fontanari, Dynamical coupling of the auroral F-region ionosphere and thermosphere: case studies, J. Atmos. Terr. Phys., 46, 625, 1984.

Aruliah, A. L., A. D. Farmer, D. Rees, and U. Brändström, The seasonal behaviour of high-latitude thermospheric winds and ion velocities observed over one solar cycle, J. Geophys. Res., 101, 15 701-15 711, 1996.

Baron, M. J., and R. H. Wand, F-region ion temperature enhancements resulting from Joule heating, J. Geophys. Res., 88, 4114-4118, 1983.

Banks, P. M., and J. R. Doupnik, A review of auroral zone electrodynamics deduced from incoherent scatter radar observations, J. Atmos. Terr. Phys., 37, 951-972, 1975.

de la Beaujardière, O., D. Alcaydé, J. Fontanari, and C. Leger, Seasonal dependence of high-latitude electric fields, J. Geophys. Res., 96, 5723-5735, 1991.

Davies, J. A., M. Lester, and I. W. McCrea, A statistical study of ion frictional heating observed by EISCAT, Ann. Geophysicae, 15, 1399-1411, 1997.

Davies, J. A., M. Lester, and I. W. McCrea, Correction to "A statistical study of ion frictional heating observed by EISCAT", Ann. Geophysicae, 16, 477-477, 1998.

Farmer, A. D., S. R. Crothers, and V. N. Davda, The winter anomaly at Tromsø, J. Atmos. Terr. Phys., 52, 561-568, 1990.

Foster, J. C., J. M. Holt, R. G. Musgrove, and D. S. Evans, Solar wind dependencies of high-latitude convection and precipitation, in Solar wind-magnetosphere coupling, Eds. Y. Kamide and J.A. Slavin, Terra/Reidel, Tokyo, 477-494, 1986.

Hapgood, M. A., M. Lockwood, G. A. Bowe, D. M. Willis, and Y. K. Tulunay, Variability of the interplanetary medium at 1 A.U. over 24 years: 1963-1986, Planet. Space Sci., 39, 411-423, 1991.

Hedin, A. E., Neutral atmosphere empirical model from the surface to the lower exosphere: MSISE, J. Geophys. Res., 96, 1159$1172,1991$.

Holt, J. M., R. H. Wand, J. V. Evans, and W. L. Oliver, Empirical models for the plasma convection at high latitudes from Millstone Hill observations, J. Geophys. Res., 92, 203-212, 1987.

Lockwood, M., and T. J. Fuller-Rowell, The modelled occurrence of non-thermal plasma in the ionospheric F-region and the possible consequences for ion outflow into the magnetosphere, Geophys. Res. Lett., 14, 371, 1987a.

Lockwood, M., and T. J. Fuller-Rowell, Correction to "The modelled occurrence of non-thermal plasma in the ionospheric F-region and the possible consequences for ion outflow into the magnetosphere", Geophys. Res. Lett., 14, 581, $1987 \mathrm{~b}$.

Lockwood, M., B. J. Bromage, R. B. Horne, J.-P. St-Maurice, D. M. Willis, and S. W. H. Cowley, Non-Maxwellian ion velocity distributions observed using EISCAT, Geophys. Res. Lett., 14, 111-114, 1987.

McCrea, I. W., M. Lester, T. R. Robinson, N. M. Wade, and T. B. Jones, On the identification and occurrence of ion frictional heating events in the high-latitude ionosphere, J. Atmos. Terr. Phys., 53, 587-597, 1991.

Moorcroft, D. R., and K. Schlegel, Evidence for non-Maxwellian ion velocity distributions in the F-region, J. Atmos. Terr. Phys. 50, 455, 1988.

Perraut, S., A. Brekke, M. Baron, and D. Hubert, EISCAT measurements of ion temperatures which indicate non-isotropic ion velocity distributions, J. Atmos. Terr. Phys., 46, 531-543, 1984.

Rees, M. H., and J. C. G. Walker, Ion and electron heating by auroral electric fields, Ann. Geophysicae, 24, 193-199, 1968.

Rishbeth, H., Thermospheric winds and the F-region: a review, J. Atmos. Terr. Phys., 34, 1-47, 1972.

Russell, C. T., and R. L. McPherron, Semiannual variation of geomagnetic activity, J. Geophys. Res., 78, 92-108, 1973.

Schunk, R. W., W. J. Raitt, and P. M. Banks, Effect of electric fields on the daytime high-latitude E- and F-regions, J. Geophys. Res., 80, 3121-3130, 1975.

St-Maurice, J.-P., and R. W. Schunk, Auroral ion velocity distributions for a polarization collision model, Planet. Space Sci., 25, 243, 1977.

St-Maurice, J.-P., and R. W. Schunk, Ion velocity distributions in the high-latitude ionosphere, Rev. Geophys. Space Phys., 17, 99 133, 1979.

St-Maurice, J.-P., W. B. Hanson, and J. C. G. Walker, Retarding potential analyser measurement of the effect of ion-neutral collisions on the ion velocity distribution in the auroral ionosphere, J. Geophys. Res., 81, 5438-5446, 1976. 